Check for updates

Cite this: RSC Adv., 2019, 9, 3994

Received 14th September 2018 Accepted 16th January 2019

DOI: 10.1039/c8ra07656a

rsc.li/rsc-advances

\section{Evaluation of novel Griess-reagent candidates for nitrite sensing in aqueous media identified via molecular fingerprint searching $\dagger$}

\author{
Linda Váradi, (D) *a Michael Breedon, ${ }^{a}$ Fiona F. Chen, ${ }^{a}$ Adrian Trinchi, ${ }^{a}$ Ivan S. Cole ${ }^{b}$ \\ and Gang Weic
}

\begin{abstract}
The Griess reaction is the most often exploited colorimetric method for the quantitative analysis of nitrite in aqueous media. The application of the currently used reagents are associated with limitations (e.g. linear response range). Herein, molecular fingerprint searching on well-known Griess-reagents was used as a tool for the identification of structurally similar, new reagent candidate molecules. Rapid and highthroughput experimental evaluation of the newly identified Griess-reagent candidates revealed that 14 of the 18 tested reagent candidates had equal or superior response displaying broader linear ranges and/or increased response gradient against various nitrite concentrations in aqueous media when compared to the parent compounds at room temperature.
\end{abstract}

\section{Introduction}

The concentration, distribution and transformation of nitrogen-species in the environment have a significant impact on nutrient supply chains, soil quality, crop yield, the atmosphere and the health of our waterways. Agricultural activities are known as the greatest contributors to the release of excess nitrogen into the environment., ${ }^{\mathbf{1} 2}$ This results in an imbalance in the nitrogen cycle causing detrimental environmental damage (e.g. algal blooms, contributing green-house gases), the treatment and prevention of which is predicted to reach about 50 billion USD p.a. by $2050 .{ }^{3}$ Within the nitrogen cycle, nitrite and nitrate are key intermediates prior to plant uptake, evaporative processes into the atmosphere, soil immobilisation as organic matter, and/or leaching into the waterways. Thus, levels of nitrate and nitrite are regularly monitored across the globe in a broad variety of analytical settings and sample types (e.g. soil, food, water, blood) via a diverse range of methods, and in almost all cases, via the subsequent measurement of nitrite before and after a reduction step that readily and quantitatively converts nitrate into nitrite. ${ }^{\mathbf{4 5}}$ Along with electrochemical, chromatographic, and capillary electrophoresis techniques, nitrite sensing applications based on optical/spectroscopic methods are the most broadly used in real-life settings. ${ }^{6}$ Especially in environmental monitoring, wet chemistry based

${ }^{a}$ CSIRO Manufacturing, Normanby Road, Clayton 3168, Australia. E-mail: linda. varadi@csiro.au

${ }^{b}$ RMIT University, GPO Box 2476, Melbourne 3001, Australia ${ }^{c}$ CSIRO Manufacturing, Bradfield Road, Lindfield 2070, Australia

$\dagger$ Electronic supplementary information (ESI) available. See DOI: 10.1039/c8ra07656a spectroscopic assays are routinely used and considered as goldstandard. This is due to the excellent specificity, selectivity, sensitivity and accuracy of these techniques along with their low-tech requirements, cost-efficiency, and ease-of-use. Nearly all of the commercially available on-field/in situ environmental nitrogen-monitoring devices ${ }^{6}$ measure nitrate and nitrite levels based on the nitrite-selective Griess reaction sequence (Scheme 1). ${ }^{7}$ These techniques employ an aromatic amine (e.g. sulfanilamide 1), that in the presence of nitrite and an acid catalyst undergo diazotisation resulting in the formation of a reactive diazonium salt 2 . This diazonium salt 2 , upon the addition of an electron rich coupling agent (e.g. $N$-(1-naphthyl)ethylenediamine, NED, 3), reacts to give a highly coloured diazo dye $\mathbf{4 .}^{\mathbf{8}}$ The absorption intensity of this dye is directly proportional to the nitrite concentration in the sample. Although rapid and reliable, the established sensing methods can be limited to narrow concentration ranges, and still require hazardous, and unstable reagents. Despite the great variety of Griess-type reagent systems that have been reported, ${ }^{\mathbf{4 , 5 , 8 , 9}}$ the discovery of reagents that require limited sample preparation, and are water

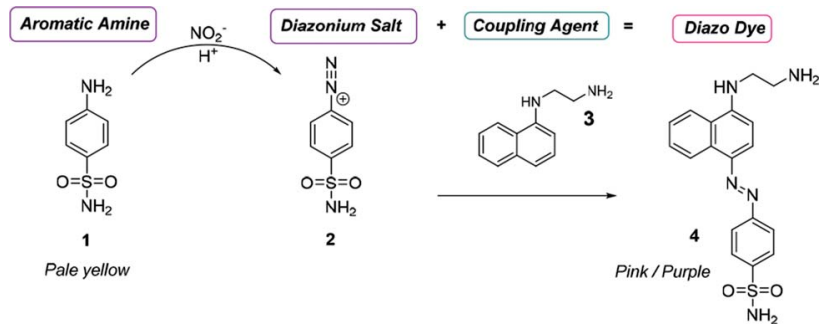

Scheme 1 Example of a Griess reaction for the detection of nitrite in aqueous media. 
soluble, non-toxic, low-cost, robust during storage have yet to be realised. Although, the multi-step Griess reaction sequence has been extensively studied for its mechanism and kinetics; ${ }^{9}$ prediction of the nitrite sensing performance of such systems is hindered due to the multiple variables known to affect the diazo-dye formation (e.g. overall kinetics, rate limiting steps, reaction conditions, incomplete pigment formation, reactivity of reagents and intermediates, optical properties of the forming pigments).

In this study molecular fingerprint searching was employed for the identification of new Griess-type reagent candidates followed by their rapid and high-throughput experimental evaluation for their nitrite sensing capability compared to some known and industrially used reagents. The novel reagent candidates were assessed at room temperature in aqueous media eliminating the requirements of $0-5{ }^{\circ} \mathrm{C}$ sensing media and the readjustment of the $\mathrm{pH}$ during the measurement. The candidates were studied in concentration ranges of 0-1000 $\mu \mathrm{M}$ relevant to environmental nutrient and contamination levels. This is equivalent to $0-46 \mathrm{ppm}$ of nitrite, the range relevant in environmental monitoring (max. $10 \mathrm{ppm}$ total $\mathrm{N}$ in drinking water by the $\mathrm{WHO},{ }^{10}$ and max. $200 \mathrm{ppm}$ in finished meat products, ${ }^{11}$ broad ranges depending on location, soil and crop types in agriculture ${ }^{\mathbf{1 2}}$ ).

\section{Experimental and methods}

\subsection{Materials and instruments}

Chemicals were purchased and used as received (purity $>98 \%$ ) from Sigma-Aldrich and Alfa Aesar. Absorption was recorded on Molecular Devices' FlexStation ${ }^{\circledR} 3$ Multi-Mode Benchtop Reader with integrated Molecular Devices' SpectraMax® ${ }^{\circledR}$ Me Microplate Reader using SoftMax® Pro v5 software. Absorption spectrum mode was used at the wavelength range of 250$750 \mathrm{~nm}$, at $24{ }^{\circ} \mathrm{C}$, with $5 \mathrm{~nm}$ steps. Greiner ${ }^{\circledR}$ Bio-One UV-Star Fbottom, chimney well $\mu$-clear 96-well plates were used.

Preparation of aromatic amine (AA) solutions. Each AA A-D (0.13 $\mathrm{mmol}$ ) was dissolved in upto $30.0 \mathrm{~mL}$ aqueous (MilliQ) $\mathrm{NaHCO}_{3}\left(10 \mathrm{~g} \mathrm{~L}^{-1}\right)$ to give $4.3 \mathrm{mM}$ stock solutions, respectively.

Preparation of NED solution. NED $\left(1.1 \mathrm{~g} \mathrm{~L}^{-1}, 4.3 \mathrm{mM}\right)$ was dissolved in MilliQ water.

Preparation of acid catalyst. $32 \mathrm{v} / \mathrm{v} \% \mathrm{HCl}(5.0 \mathrm{~mL})$ was diluted with MilliQ water $(30.0 \mathrm{~mL})$.

Preparation of $\mathrm{NaNO}_{2}$ solution. $\mathrm{NaNO}_{2}(276 \mathrm{mg} / 10 \mathrm{~mL}$ in MilliQ water) stock solution was prepared and made into 100times dilution $(2.76 \mathrm{mg} / 10 \mathrm{~mL})$ to reach $4.0 \mathrm{mM}$ concentration. From the $4.0 \mathrm{mM}$ solution, standards of 4.0, 2.0, 1.0, 0.5, 0.25, $0.125,0.0625 \mathrm{mM}$ were prepared by double dilution.

\subsection{Methods}

Molecular fingerprint searching for the selection of new reagent candidates. As an alternative to intuitive reagent selection, here, we investigate the application of computerassisted molecular fingerprint searching for the identification of new Griess-reagent candidates. This tool is routinely and reliably used, for example, in medicinal chemistry for the unbiased generation of hit lists based on structural similarity of drug molecules. ${ }^{\mathbf{1 3 , 1 4}}$ Here, four well-known aromatic amines (4nitroaniline A, sulfanilic acid B, 4-aminobenzoic acid C, and 2aminobenzoic acid D) were chosen as parent compounds (input). New candidates (output) were generated through similarity comparisons of molecular fingerprints between the parent compounds (input) and the entries within the PubChem archive, which hosts over 23 million compounds. ${ }^{15}$ Molecular fingerprinting is a powerful technique to generate a machine readable representation of molecules in the form of binary strings, as represented in Fig. S1. $\dagger$ Each position in this binary string represents a defined piece of structural information of the molecule (e.g. chemical elements, ring and bond configurations). (A comprehensive list of the structural descriptors can be found at http://pubchem.ncbi.nlm.nih.gov/.) The binary string was then compared between the parent compound and each of the PubChem compounds. By defining the ratio between the number of common bits in the fingerprints over the number of remaining bits within the two fingerprints, a Tanimoto index (similarity) was generated ranging from 0 to $1 .^{13}$ The Tanimoto index is an effective way of quantifying the structural similarity between two chemical compounds and is suitable for general purpose molecular similarity searching without any prior knowledge of the searched chemical structures. A higher index implies that more bits are identical in the compared fingerprints, hence, the greater the structural similarity is. For a given parent compound (A, B, C, D), molecules from the PubChem database were sorted by the Tanimoto index from the most to the least similar, these were considered as possible new candidates. Given the intractable number of potential validation experiments, the following selection rules were applied to narrow the candidate molecules for experimental evaluation. Only candidates that fulfilled the following selection criteria have been tested (for an example of the selection process see Table $\mathrm{S} 1, \dagger$ for the selected candidates see Table 1);

(i) Greater than $80 \%$ structural similarity which fall within the top 100 rank of the output list,

(ii) Presence of primary amine substitution (essential for diazotization) on the aromatic ring,

(iii) Absence of heavy metals,

(iv) Water solubility in the target concentration range,

(v) Various salt forms of the same core compounds were considered as equivalent,

(vi) Commercial availability.

The selected candidates subjected to experimental evaluation were A1-3 for parent compound A, B1-3 for parent compound B, C1-4 for parent compound C, and D1-D8 for parent compound $\mathbf{D}$ (Table 1). The coupling agent NED 3 was selected as a fixed variable in this study.

High-throughput UV-Vis absorption assay to identify new candidates with extended linear range. Both the parent compounds and new candidates were subjected to highthroughput screening for their diazo-dye formation and absorption profiles at various nitrite concentrations under previously reported Griess reaction conditions. ${ }^{16,17}$ As a general procedure, in a 96-well plate, $50 \mu \mathrm{L}$ of the aqueous solution of 
Table 1 Absorption maxima of diazo-dye formed from aromatic amines $A-D$, linear range $(\mu M)$, and sensing sensitivity within the linear range, and the toxicity of the aromatic amines as assessed by GSH safety data sheets. Parent compounds A, B, C and D and new candidates A1-3, B1-3, C1-4, D1-8. [Sensit. = sensitiser; Corr. = corrosive; non-tox = non-toxic]

\begin{tabular}{|c|c|c|c|c|c|c|c|}
\hline No. & Name of compound & Tanimoto index & $\begin{array}{l}\text { (i) Diazo-dye } \\
\lambda_{\max }(\mathrm{nm})\end{array}$ & $\begin{array}{l}\text { (ii) Linear } \\
\text { range }(\mu \mathrm{M})\end{array}$ & $\begin{array}{l}R^{2} \text { within } \\
\text { linear range }\end{array}$ & $\begin{array}{l}\text { (iii) Gradient } \\
\text { of linear range }\end{array}$ & (iv) Toxicity \\
\hline $\mathbf{A}$ & 4-Nitroaniline & 1.00 & 560 & $0-31.25$ & 0.9990 & 0.0250 & Cat 3 \\
\hline $\mathbf{A 1}$ & 3-Nitroaniline & 0.93 & 535 & $0-31.25$ & 0.9986 & 0.0268 & Cat 3 \\
\hline $\mathbf{A 2}$ & 2-Nitroaniline & 0.91 & 550 & $0-125$ & 0.9987 & 0.0196 & Cat 4 \\
\hline $\mathbf{A 3}$ & 2-Fluoro-5-nitroaniline & 0.89 & 550 & $15.62-250$ & 0.9924 & 0.0038 & Irritant \\
\hline B & Sulfanilic acid & 1.00 & 525 & $0-125$ & 0.9869 & 0.0207 & Sensit. \\
\hline B1 & 3-Aminobenzenesulfonic acid & 0.94 & 530 & $0-125$ & 0.9947 & 0.0179 & Irritant \\
\hline \multirow[t]{2}{*}{ B2 } & 2-Aminobenzenesulfonic acid & 0.94 & 545 & $0-31.25$ & 0.9973 & 0.0193 & Corr. \\
\hline & & & & $125-1000$ & 0.9904 & 0.0014 & \\
\hline B3 & 2,4-Diaminobenzenesulfonic acid & 0.90 & 535 & $0-125$ & 0.9418 & 0.0004 & Irritant \\
\hline $\mathbf{C}$ & 4-Aminobenzoic acid & 1.00 & 535 & $0-125$ & 0.9962 & 0.0213 & Sensit. \\
\hline C1 & 3-Aminobenzoic acid & 0.91 & 525 & $0-125$ & 0.9983 & 0.0200 & Cat 4 \\
\hline $\mathbf{C 2}$ & 4-Aminophthalic acid & 0.90 & 535 & $0-125$ & 0.9942 & 0.0203 & Irritant \\
\hline C3 & Methyl 4-aminobenzoate & 0.89 & 540 & $0-125$ & 0.9958 & 0.0207 & Non-tox \\
\hline C4 & 3-Fluoro-4-aminobenzoic acid & 0.97 & 525 & $0-125$ & 0.9963 & 0.0212 & Cat 4 \\
\hline D & 2-Aminobenzoic acid & 1.00 & 545 & $0-125$ & 0.9947 & 0.0085 & Irritant \\
\hline D1 & 2-Amino-3-fluorobenzoic acid & 0.95 & 525 & $0-125$ & 0.9995 & 0.0191 & Cat 4 \\
\hline D2 & 2-Amino-4-fluorobenzoic acid & 0.97 & 530 & $0-125$ & 0.9961 & 0.0170 & Irritant \\
\hline D3 & 2-Amino-5-fluorobenzoic acid & 0.97 & 545 & $0-125$ & 0.9940 & 0.0105 & Irritant \\
\hline D4 & 2-Amino-6-fluorobenzoic acid & 0.96 & 535 & $0-125$ & 0.9991 & 0.0187 & Irritant \\
\hline D5 & 2-Amino-4,5-difluorobenzoic acid & 0.95 & 535 & $0-62.5$ & 0.9880 & 0.0150 & Irritant \\
\hline D6 & 2-Amino-3-methylbenzoic acid & 0.92 & 535 & $0-125$ & 0.9904 & 0.0005 & Cat 4 \\
\hline D7 & 2-Amino-5-methylbenzoic acid & 0.93 & 560 & $0-125$ & 0.9937 & 0.0015 & Irritant \\
\hline D8 & 2-Amino-6-methylbenzoic acid & 0.92 & 545 & $0-125$ & 0.9985 & 0.0179 & Irritant \\
\hline
\end{tabular}

each of the selected aromatic amines was mixed at room temperature with $50 \mu \mathrm{L}$ of aqueous $\mathrm{HCl}$ acid catalyst. Then 50 $\mu \mathrm{L}$ of the corresponding $\mathrm{NaNO}_{2}$ solution was added. After 2 minutes resting at room temperature to ensure diazonium salt formation, $50 \mu \mathrm{L}$ of the coupling agent, NED was added resulting in a final nitrite concentration in the $0-1000 \mu \mathrm{M}$ range. The well-plates were shaken for 5 seconds prior to obtaining the UV-Vis absorption spectra on the FlexStation ${ }^{\circledR} 3$ plate reader, and scans were recorded in duplicates between 250 and $750 \mathrm{~nm}$. For each candidate, absorption intensity as a function of wavelength was plotted at various nitrite concentrations (not included here). Then, absorption intensities versus various nitrite concentrations were plotted at the absorption maxima corresponding to the forming diazo dyes, respectively, to identify linear response range and gradient of the linear range (Table 1 and Fig. 1).

\section{Results and discussion}

\subsection{Absorption characteristics of the formed diazo dyes}

For the tested new candidates, the formation of the corresponding diazo dyes (representative to the presence of nitrite) have resulted in the rise of characteristic absorption peaks with maxima between 525 to $560 \mathrm{~nm}$ (Table 1).

The reaction steps, which lead up to the formation of diazo dye, can also result in various by-products that subsequently contribute to the absorption profile of these aqueous mixtures. ${ }^{8,9}$ The relative ratio of the forming species, as well as the absorption characteristics of the signal producing diazo dyes are both affected by the electron distribution (defined by the substituent pattern) of the Griess-reagents used. When the diazo dyes are isolated in their chemically pure form, these relations can be readily assessed. Under the applied assay conditions in this study, candidates D6 and D7 showed insignificant absorption change presumably due to the lack of diazo dye-formation. For all other candidates, increasing nitrite concentrations resulted in the initial increase of the absorption intensities at the $\lambda_{\max }$ of the diazo dyes forming until, in most cases (apart from B2), saturation was reached prior to declining absorption intensities upon further increase in the amount of nitrite present (Fig. 1). This is presumably due to the occurring side reactions (e.g. destabilisation and hydrolysis of diazonium salt, hence lack of diazo dye formation) that are known to 

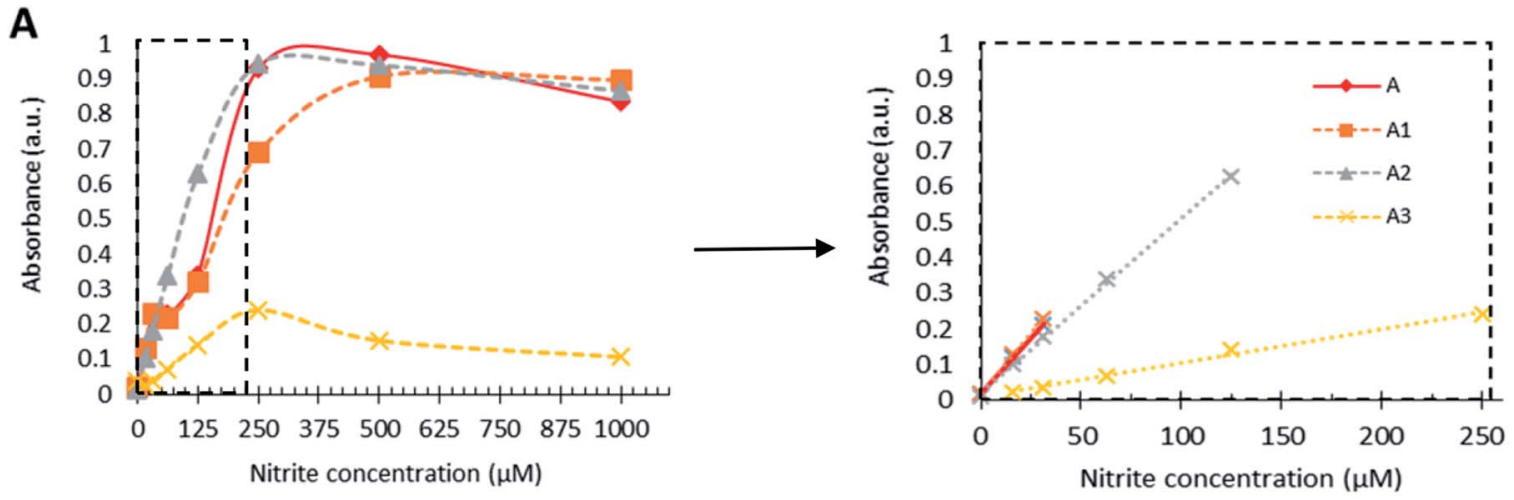

B
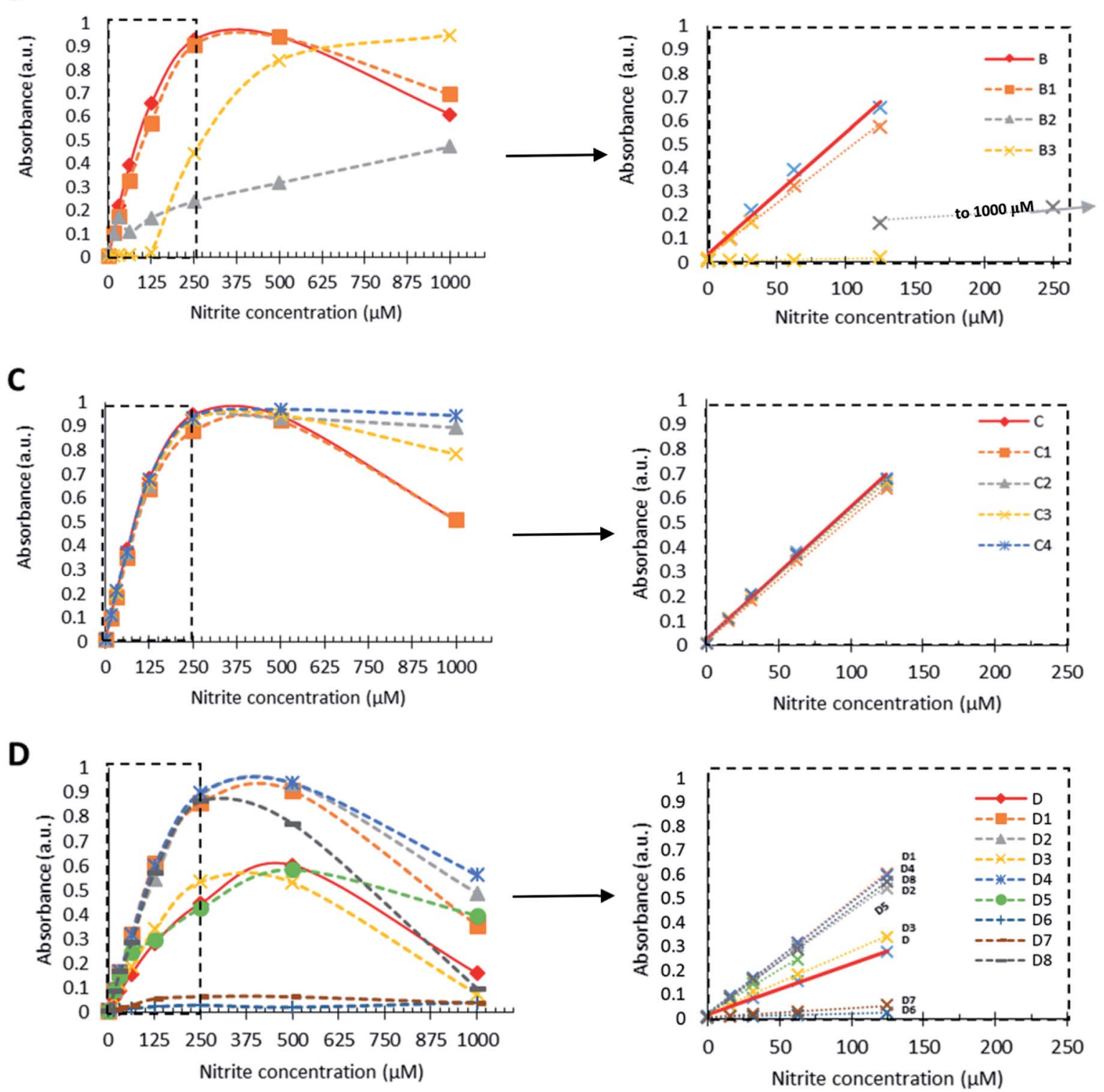

Fig. 1 Absorption intensities as a function of nitrite concentration for each parent compound and their corresponding new candidates. Comparison of linear ranges and sensitivity of parent compounds (red solid line) and evaluated candidate compounds (dashed line).

become more prevalent at higher nitrite concentrations., ${ }^{9,18}$ Moreover, across the various candidates, the absorption intensities displayed relative to the concentration of nitrite also depends on the molar extinction coefficient $(\varepsilon)$ of each of the in situ formed diazo dyes. As a general principle, the presence of electron withdrawing groups (EWG) in ortho/para position to 


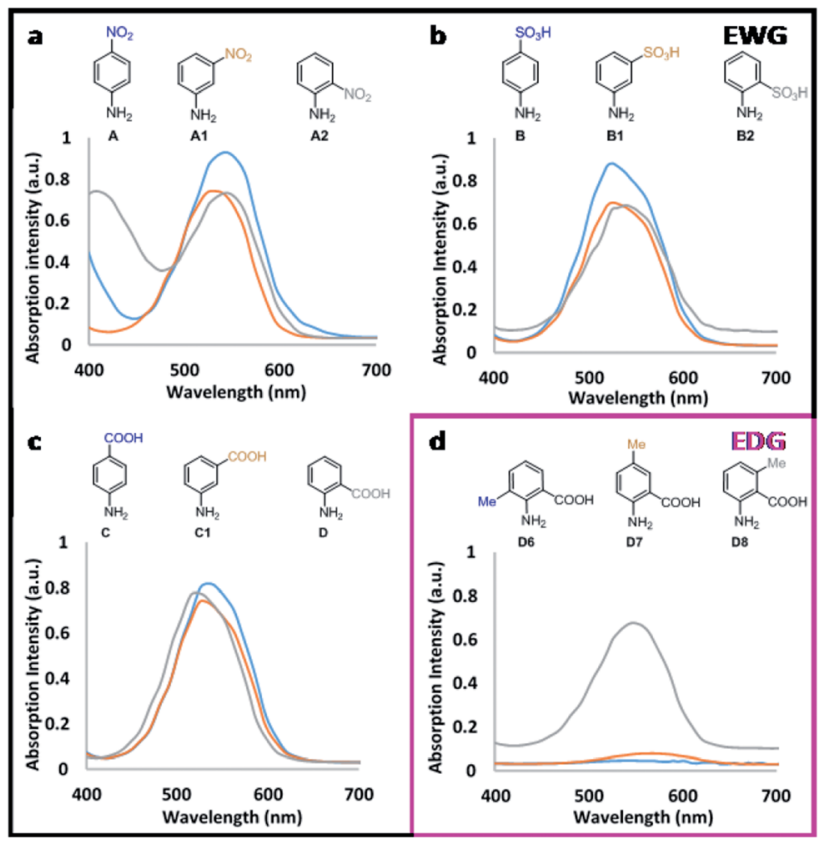

Fig. 2 Substituent patterns and absorption responses at $31.25 \mu \mathrm{M}$ nitrite concentration obtained from amines (a) A (blue), A1 (orange), A2 (grey); (b) B (blue), B1 (orange), B2 (grey); (c) C (blue), C1 (orange), D (grey); (d) D6 (blue), D7 (orange), D8 (grey).

the reaction centre (here: amino group) is expected to undergo nucleophilic attack by the coupling agent more readily than the meta derivative as a result of lower electron density on the diazonium group. However, within our dataset, the observed absorption intensities stayed largely unaffected by the position of EWGs (Fig. 2). For example para-, meta- and ortho-nitroaniline $\mathbf{A}, \mathbf{A 1}$, and $\mathbf{A 2}$, respectively, gave practically overlapping response curves at identical nitrite concentrations of $31.25 \mu \mathrm{M}$ (Fig. 2a). This is presumably due to the rapid formation of the diazonium ions to yield the sensing molecule diazo-dye. On the contrary, the position of electron donating groups (EDG) had a major effect on dye formation and colour response. As an example, between methyl substituted candidates D6-8, metamethyl-2-aminobenzoic acid D8 produced coloured signal with much higher absorption intensity than the ortho and para isomers (Fig. 2d). Thus, ortho/para EDGs are presumed to hinder the diazo-dye formation by providing more stability to the intermediate diazonium ion.

\subsection{Nitrite sensing capability of new candidates relative to their corresponding parent compounds based on high- throughput assay}

To compare the nitrite sensing performance of all tested compounds, the following numerical descriptors were taken into account (Table 1, Fig. 1):

(i) Absorption maxima for each tested compound were within 525-560 nm range, as discussed above, thus considered equally detectable via spectroscopic methods in real-life applications.

(ii) Linear range of the absorption intensity as a function of nitrite concentration (with $R^{2}>0.98$ ) is considered ideal when covering a broad range of nitrite concentration. Most of the tested candidates displayed good linearity in the $0-125 \mu \mathrm{M}$ range, with some exceptions of narrower or wider applicable sensing range.

(iii) Gradient of the fitted linear regression was taken as a measure of reliable and sensitive response to changes in nitrite concentration. The greater the gradient, the more superior the candidate is considered. Lastly, as essential for real-life applicability.

(iv) Associated hazard levels can also be considered.

Firstly, for each parent compound, these measures of nitrite sensing performance was evaluated within the group of tested candidates.

Parent compound 4-nitroaniline A showed linear response (with $R^{2}=0.999$ ) up to $31.25 \mu \mathrm{M}$ dissolved nitrite; as did candidate A1, while 2-nitroaniline $\mathbf{A 2}$ and 2-fluoro-5nitroaniline $\mathbf{A} 3$ outperformed the parent compound. 2-Nitroaniline $\mathbf{A} 2$ had essentially equal gradient (sensitivity) as $\mathbf{A}$, but within a greatly extended linear concentration range of $0-125$ $\mu \mathrm{M}$. 2-Fluoro-5-nitroaniline $\mathbf{A 3}$, although less sensitive to changes in nitrite concentration, showed potential to cover a wide concentration range of 15.625-250 $\mu \mathrm{M}$ with comparable linear regression to parent compound A. Thus, dual use of $\mathbf{A 3}$ and A2 (incorporated into a test kit) could allow for broad concentration-range screening by $\mathbf{A} \mathbf{3}$, followed by more precise quantitation by $\mathbf{A} \mathbf{2}$ if and when nitrite is present in smaller quantities. Additionally, both candidate $\mathbf{A 2}$ and $\mathbf{A 3}$ have significantly lower health and environmental hazards compared to 4-nitroaniline A, which is a 'Category 3' acutely toxic reagent.

Sulfanilic acid $\mathbf{B}$ is the most widely used Griess-reagent among the parent compounds assessed in this study. Under strict assay-temperature control $\left(0-5^{\circ} \mathrm{C}\right) \mathrm{B}$ can be used reliably; however at room temperature its linear regression $\left(R^{2}=0.9869\right)$ within the $0-125 \mu \mathrm{M}$ nitrite concentration range is outperformed by 3-aminobenzenesulfonic acid B1 $\left(R^{2}=0.9947\right)$ displaying equal gradient of absorption response. Combined with the 30-fold lower price and low toxicity when compared to parent compound $\mathbf{B}$, the comparable sensing performance makes B1 a preferable nitrite sensing molecule. 2-Aminobenzenesulfonic acid $\mathbf{B} 2$ displayed the broadest dynamic range among all the assessed candidates at the tested concentrations with neither detector saturation, nor declining absorbance observed at high concentrations of nitrite. Below $31.25 \mu \mathrm{M}$, B2 responded to nitrite with equal gradient (sensitivity) to that of parent compound B. More significantly, B2 displayed linear response to changes in nitrite concentration over a much wider, 125-1000 $\mu \mathrm{M}$ concentration range, albeit with a less distinguished optical response (lesser gradient). 2,4-Diaminobenzenesulfonic acid B3 displayed insignificant colour response, presumably due to the presence of a second amino group, which is also prone to reaction under the test conditions.

The tested derivatives of 4 - and 2-aminobenzoic acid, $\mathbf{C}$ and D respectively, displayed linear response to nitrite concentrations up to $125 \mu \mathrm{M}$. All candidates proposed for 4 -aminobenzoic acid $\mathbf{C}$ exhibited practically identical sensing capabilities. Interestingly, the position of the moderately EWG carboxylic group, or the presence of electronegative (but weakly 


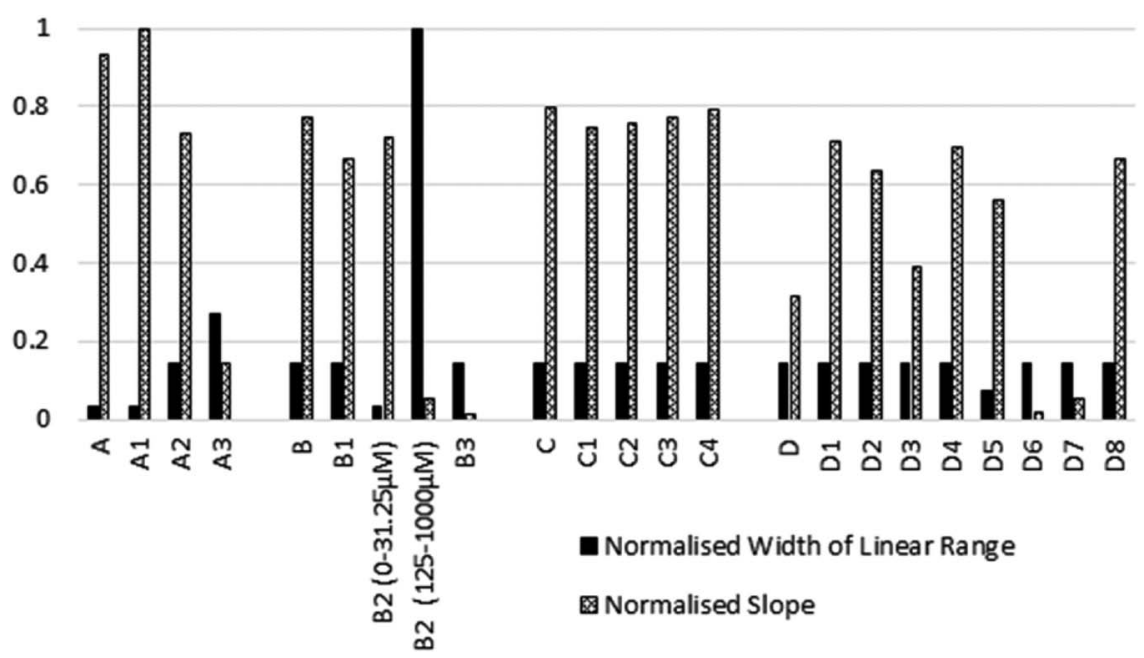

Fig. 3 Normalised values - within the tested parent compounds and candidates - of the width of the linear absorption response range as function of nitrite concentration (black solid bars); and the gradient of the linear regression within this linear range (dashed bars).

deactivating) fluorine, did not influence the measured intensity. However, as discussed above, EDG (activating) methyl groups at positions ortho and para to the reaction centre amine have, presumably, suppressed the formation of coloured diazo dyes (D6, D7). Moreover, all fluorinated candidates yielded a greater gradient of the responses, thus exhibiting increased sensitivity over the non-fluorinated compounds.

A comparison of the normalised width of the linear range and the gradient of the sensing signal across all of the tested reagent combinations are presented in Fig. 3, and Table S2. $\dagger$ Width of the linear range showed to be outstanding for candidates $\mathbf{B} 2$ and $\mathbf{A 3}$, followed by the mostly equivalent performing candidates, with D5, A and A1 limited to narrow concentration ranges. In terms of gradients within the linear range, $\mathbf{A}$ and $\mathbf{A 1}$ showed excellent sensitivity. The rest of the candidates and parent compounds performed rather evenly when response gradients were considered, apart from A3, B2, B3, D, D3, D6, and $\mathbf{D} 7$, which had less prominent colour intensity changes upon changes of nitrite concentration. For ideal overall sensing performance, sufficiently wide linear range and relatively greater gradient are concurrently desired. This combined criteria was best met by A2, A3, C1-C4, D1-D4, and D8 within the assessed set of reagents.

\section{Conclusions}

In summary, computer assisted molecular fingerprint searching facilitated the unbiased identification of alternative Griessreagents from a large ( $>23 \mathrm{M}$ compound) data base. The output list of potential candidates created by this chemical informatics method is somewhat limited in diversity due to the similarity matching nature of molecular fingerprint searching. This output list was further narrowed in scope by setting simple and practical selection rules. It is also important to note, that the relation between structural similarity and colorimetric response similarity is not accounted for in such methodologies. However, especially in the design of novel Griess-type reagent systems where a multi-step reaction occurs involving intermediates with unknown variety and stability; an unbiased and calculated molecular similarity can complement intuitive reagent selection. It was found that 14 out of the 18 evaluated candidates showed similar or superior nitrite sensing potential when compared to their corresponding parent compounds. Candidates A2, A3, and B2 displayed extended linear ranges, meanwhile D1-4 and D8 were calculated to have steeper gradients, hence are more sensitive to changes in nitrite concentration than their corresponding parent compounds. Meanwhile, normalised values across all tested reagents showed that 11 new candidates met the required combined performance measures (linearity and signal strength) to meet or outperform the parent compounds. Thus, the proposed combination of molecular fingerprint searching and rapid experimental evaluation - as demonstrated on this particular Griess reaction - offers a methodology for reagent discovery.

\section{Conflicts of interest}

There are no conflicts to declare.

\section{Notes and references}

1 E. P. A. (USA), Inventory of U.S. Greenhouse Gas Emissions and Sinks: 1990-2015, 2015.

2 P. Kumar, K. H. Kim, V. Bansal, T. Lazarides and N. Kumar, J. Ind. Eng. Chem., 2017, 54, 30-43.

3 A. G. Good and P. H. Beatty, PLoS Biol., 2011, 9, e1001124.

4 M. J. Moorcroft, J. Davis and R. G. Compton, Talanta, 2001, 54, 785-803.

5 Q.-H. Wang, L.-J. Yu, Y. Liu, L. Lin, R.-g. Lu, J.-p. Zhu, L. He and Z.-L. Lu, Talanta, 2017, 165, 709-720.

6 T. M. Schierenbeck and M. C. Smith, Environ. Sci. Technol., 2017, 51, 4755-4771.

7 A. R. Jalalvand, M. Mahmoudi and H. C. Goicoechea, RSC $A d v .$, 2018, 8, 23411-23420.

8 V. M. Ivanov, J. Anal. Chem., 2004, 59, 1002-1005.

9 J. B. Fox, Anal. Chem., 1979, 51, 1493-1502. 
10 WHO, Guideline for Nitrate and Nitrite in Drinking-water, 2011.

11 FDA, Code of Federal Regulations, Food and Drugs - Food Additives permitted for direct addition to food for human consumption, https://www.accessdata.fda.gov/scripts/cdrh/ cfdocs/cfCFR/CFRSearch.cfm?fr=172.175, 2003.

12 B. C. T. Macdonald, Y. F. Chang, A. Nadelko, S. Tuomi and M. Glover, Soil Res., 2017, 55, 264-272.

13 D. Bajusz, A. Rácz and K. Héberger, J. Cheminf., 2015, 7, 20.
14 G. Maggiora, M. Vogt, D. Stumpfe and J. Bajorath, J. Med. Chem., 2014, 57, 3186-3204.

15 W. Moore, R. Talati, P. Bhattacharji and T. Bilfinger, J. Vasc. Interv. Radiol., 2015, 26, 312-319.

16 Promega Corporation, Technical Bulletin, Griess Reagent System G2930, 2009, part\# TB229.

17 Molecular Probes Inc., Griess reagent kit for nitrite determination (G-7921, MP07921), 2003.

18 D. Tsikas, J. Chromatogr. B, 2007, 851, 51-70. 